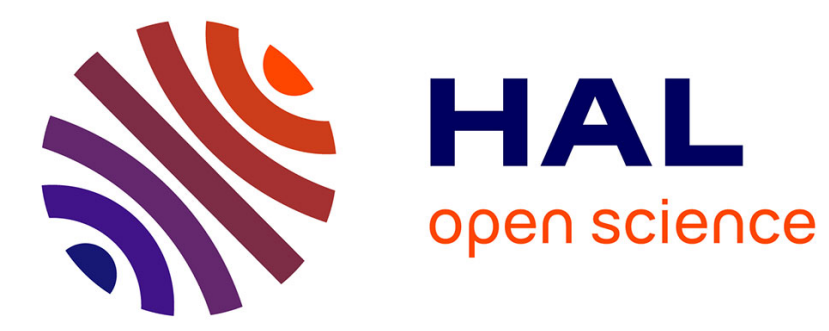

\title{
Two-photon absorption in low-dimensional heterostructures
}

R. Cingolani, M. Lepore, R. Tommasi, I. Catalano, H. Lage, D. Heitmann, K. Ploog

\section{- To cite this version:}

R. Cingolani, M. Lepore, R. Tommasi, I. Catalano, H. Lage, et al.. Two-photon absorption in lowdimensional heterostructures. Journal de Physique IV Proceedings, 1993, 03 (C5), pp.C5-131-C5-138. 10.1051/jp4:1993523 . jpa-00251609

\section{HAL Id: jpa-00251609 https://hal.science/jpa-00251609}

Submitted on 1 Jan 1993

HAL is a multi-disciplinary open access archive for the deposit and dissemination of scientific research documents, whether they are published or not. The documents may come from teaching and research institutions in France or abroad, or from public or private research centers.
L'archive ouverte pluridisciplinaire HAL, est destinée au dépôt et à la diffusion de documents scientifiques de niveau recherche, publiés ou non, émanant des établissements d'enseignement et de recherche français ou étrangers, des laboratoires publics ou privés. 


\title{
Two-photon absorption in low-dimensional heterostructures
}

\author{
R. CINGOLANI ${ }^{(1)}$, M. LEPORE, R. TOMMASI, I.M. CATALANO, H. LAGE*, \\ D. HEITMANN ${ }^{*}(2)$ and K. PLOOG ${ }^{*}(3)$
}

Unità GNEQP, Dipartimento di Fisica, Università di Bari, Via Amendola 173, 70110 Bari, Italy

${ }^{*}$ Max-Planck-Institut für Festkörperforschung, Heisenbergstrasse 1, 7000 Stuttgart 80, Germany

\begin{abstract}
We briefly review the physics of two-photon absorption processes in lowdimensional systems. The anisotropic selection rules of the multiphoton absorption in quantum wells and quantum wires are exploited to study the 2 p-exciton states and the otherwise forbidden transitions among quantized subbands of different quantum numbers.
\end{abstract}

\section{Introduction}

The non-linear optical properties of low-dimensional semiconductors have attracted great interest in the last years. In particular, two-photon absorption (TPA) has been investigated in detail in semiconductor quantum wells, both experimentally and theoretically, to elucidate the impact of the reduced dimensionality on the non-linear absorption processes [1]. Currently, interest has turned to systems with even lower dimensionality, like $1 \mathrm{D}$ quantum wires and $0 \mathrm{D}$ quantum dots (for a review see ref. [2]), whose excitonic non-linearities are expected to be stronger than in bulk or $2 \mathrm{D}$, due to the modification of the density of states and to the larger overlap of the electron and hole wavefunctions. The two-photon absorption process can be considered as the fundamental absorption mechanism for excitation in the transparency region of a crystal . As schematically depicted in Fig.1, the simultaneous absorption of two photons of energies $\hbar \omega_{1} \leq E_{g}$ and $\hbar \omega_{2} \leq E_{g}$ produces an electron-hole pair. The final state of this process is usually an exciton which decays within its lifetime. In the recombination some luminescence is emitted, whose intensity depends quadratically on the intensity of the exciting radiation. The calculation of the TPA transition rate $\left(W_{c v}\right)$ is performed in the frame of the perturbation theory, assuming that the absorption transition involves a virtual intermediate state $[3,4,5,6,7]$. The TPA transition rate is usually calculated within a two-band scheme in which the summation over all the intermediate states are replaced by a single virtual state. The intermediate virtual state is assumed to be coincident with either the initial or the final state and the total TPA process

(1) Permanent address: Dipartimento Scienza dei Materiali, Università di Lecce, Via Arnesano, 73100 Lecce, Italy

(2) Permanent address: Institut fuer Agewandte Physik, Jungenstr. 11, 2000 Hamburg, Germany

(3) Permanent address: Paul Drude Institut fuer Festkoerperelektronik,1086 Berlin, Germany 
occurs via an intra-valence or intra-conduction subband transition (circular arrow in Fig.1). The basic result of the theories is that the TPA rate in low-dimensional systems is strongly anisotropic. This anisotropy comes from the dependence of the matrix elements on the polarization direction of the exciting electromagnetic field with respect to the carrier confinement direction. In quantum wells the confinement direction is along the $\mathrm{z}$-axis, whereas the quantum wires have an additional lateral confinement along the y-axis. In quantum wells, when the light is polarized perpendicular to the confinement direction, the matrix elements of the momentum operator are nonvanishing only for transitions involving states with the same principal quantum number $\mathrm{n}$. The selection rule $\Delta n=0$ is therefore expected to be valid for the case $\epsilon \perp z$. Conversely, TPA transitions for the polarization $\epsilon \| z$ can only involve subbands whose quantum numbers differ by an odd integer $(\Delta n \neq 0$ selection rule) and light-hole valence subbands.

In quantum wires the TPA matrix elements are no longer identical on the basal xy plane, due to the additional lateral confinement. In this case, optical transitions between quantum wire states with the same quantum numbers $\left(\Delta n_{y}=0\right)$ are allowed when the polarization direction of the exciting beam is perpendicular to the wire quantization direction $(\epsilon \perp y)$, whereas $\Delta n_{y}= \pm 1$ transitions are dominant in the $\epsilon \| y$ geometry. Inclusion of the excitonic states in the calculation of the TPA rate leads to selection rules complementary to the ones usually assumed for the linear excitonic absorption, i.e. TPA transitions with $\Delta \mathrm{n}=0$ selection rule involve final excited 2p excitonic states, while 1s states, allowed in the linear absorption processes, become forbidden in the TPA processes. On the other hand $\Delta n=1$ TPA transitions are allowed only with 1 s final excitonic states. The above selection rules come out from the requirements of parity conservation in the total TPA process.

\section{Experimental}

Due to the complementary selection rules, two-photon spectroscopy offers the unique possibility to access directly $2 p$ excitonic states. Since the diameter of the $2 \mathrm{p}$ excited states is enlarged by a factor 4 (valid for $3 \mathrm{D}$ excitons) with respect to is excitons, these states should be effectively quantized and therefore exhibit a true lower dimensional character even in relatively wide quantum wires and quantum wells. In the last years several experimental studies have been reported on TPA spectroscopy in $\mathrm{GaAs} / A l_{x} G a_{1-x}$ As multiple quantum wells $[8,9,10,11,12,13,14]$ and on GaAs quantum wires [15 ]. The basic purpose of these experiments was the investigation of the TPA absorption processes in resonance with quantized subbands. It should be mentioned that in contrast to the case of bulk crystals, the experimental investigation of TPA processes in low dimensional heterostructures is difficult because very weak absorption changes through the ultra-small volume of the heterostructure have to be detected

In this work we concentrate on TPA experiments performed on MBE grown GaAs/AlGaAs quantum wells and on quantum wires fabricated by holographic 
patterning and subsequent reactive ion etching of the quantum wells. The quantum well structure consisted of $25 \mathrm{GaAs}$ wells of width $L_{z}=106 \AA$ and $A l_{0.36} G a_{0.64} A s$ barriers of width $L_{b z}=153 \AA$ grown on top of a $1 \mu \mathrm{m}$ thick $A l_{0.36} G a_{0.64} A s$ cladding layer providing optical confinement of the luminescence. The processed quantum well structure resulted in a regular array of quantum wires with $280 \mathrm{~nm}$ periodicity and a crystalline wire width $L_{y}=60 \pm 5 \mathrm{~nm}$, as determined by the analysis of the high resolution $x$-ray diffraction spectra. Details on the structural and linear optical properties of the sample are reported in Ref.[16]. The non-linear absorption has been studied by measuring the TPA-induced photoluminescence excitation spectra at $10 \mathrm{~K}$ for different polarization directions of the exciting laser beam. The detection energy was set at the fundamental exciton state (around $E_{11}^{1 s}=1.554 \mathrm{eV}$ for the well and the wires), independently measured by linear PLE spectroscopy, and by scanning the exciting photon energy in the transparency region of the crystal. Details on the experimental equipement are given in ref.[11]. The quadratic behavior of the detected luminescence signal versus the excitation intensity was checked at each experimental point and several measurement runs were carried out at all exciting wavelenghts. Under the adopted experimental conditions, the signal to noise ratio was of the order of $200: 1$.

\section{Results and Discussion}

In Fig. 2 we show the typical intensity dependence of the nonlinear luminescence induced by the absorption of two photons of energy $\hbar \omega=0.795 \mathrm{eV}(2 \hbar \omega=1.590 \mathrm{eV})$ and $\hbar \omega=0.805 \mathrm{eV}(2 \hbar \omega=1.610 \mathrm{eV})$ in the GaAs/ AlGaAs quantum wire array. As expected for the TPA process, the emission intensity grows quadratically with the exciting power density. A similar behavior is obtained at all exciting wavelengths in the TPA experiments on quantum wells and quantum wires discussed in this work.

In fig. 3 we show the one photon absorption PLE (OPA-PLE) and the polarization dependent TPA-PLE measured in the GaAs/AlGaAs multiple quantum wells. The OPA-PLE curve exhibits the heavy and light hole exciton peaks associated with the $n=2$ subbands in the well and two weaker parity allowed transitions $\left(E_{13 h}\right.$ and $\left.E_{24 h}\right)$. In addition, a sharp $E_{21 l}$ transition is observed at $1.664 \mathrm{eV}$. The interpretation of the OPA-PLE spectrum has been made according to the well known envelope function model, assuming non-parabolic masses and 60:40 conduction to valence band offset ratio (in this experiments the investigation was not extended to lower energies because the $E_{11 l}$ line was very close to the detection wavelength and could not be resolved in the PLE spectrum due to straight-light rejection problems).

The TPA-PLE spectra recorded in the $\epsilon \perp z$ and $\epsilon \| z$ configurations are displayed in Figs. 3b and 3c. The $\epsilon \perp z$ geometry exhibits $\Delta n=0$ excitonic peaks. The structures labeled $E_{22 h}^{2 p}$ and $E_{22 l}^{2 p}$ are blue shifted by about $8 \mathrm{meV}$ with respect to the corresponding $1 \mathrm{~s}$ states observed in the linear spectrum. This is consistent with the expected $2 p$-1s splitting of the heavy and light-hole excitons associated 
with the $\mathrm{n}=2$ subband in a $10 \mathrm{~nm}$ GaAs $\mathrm{QW}$ [17]. Systematic measurements on quantum wells of different well widths, allowed us to evaluate a $2 \mathrm{p}$ exciton binding energy of $1.5 \mathrm{meV}$ for the $10 \mathrm{~nm}$ well, and of $2 \mathrm{meV}$ for a $6 \mathrm{~nm}$ well.

Dramatic changes in the TPA-PLE spectrum occur in the $\epsilon \| z$ configuration. This curve exhibits sharp $\Delta \mathrm{n}=1$ excitonic resonances labeled $E_{12 l}$ and $E_{21 l}$ (at $1.631 \mathrm{eV}$ and $1.664 \mathrm{eV}$, respectively) and a broad structure around $1.652 \mathrm{eV}$. The $E_{12 l}$ and $E_{21 l}$ transitions are forbidden in the OPA process, and can only be observed in TPA spectroscopy in the $\epsilon \| z$ configuration. In addition, the $E_{22 h}^{2 p}$ and $E_{22 l}^{2 p}$ transitions observed in the $\epsilon \perp z$ configuration disappear, indicating a large on/off ratio of the observed non-linearity. According to the parity selection rules given in Tab.2, the $E_{12 l}^{1 s}$ peak is related to the $1 \mathrm{~s}$ exciton associated to the $\mathrm{n}=1$ electron subband and to the $\mathrm{n}=2$ light-hole subband.

The attribution of the $E_{21 l}$ transition observed at the same energy in both the linear and non-linear spectra of Fig.3 is not straightforward. Probably, this is due to the Coulomb coupling between excitons belonging to different subbands which enhances the oscillator strength of the $2 \mathrm{p}$ state of the $E_{21 l}$ exciton at well widths of the order of $10 \mathrm{~nm}$. A possible role of the $3 \mathrm{~d}$ excited state of the $E_{21 h}$ exciton is also indicated at the broad bump of Fig.3b.

The OPA-PLE and TPA-PLE spectra of the quantum wires are displayed in Fig.4 . Before entering into a detailed discussion of the TPA-PLE data we briefly summarize the results of the linear spectroscopy of quantum wires under lowand high-excitation intensity. Due to the relatively large width of the investigated quantum wires, which amounts approximately to four times the bulk exciton Bohr radius $\left(a_{o}\right)$, the quantum effects induced by the lateral confinement (y-direction) are expected to be different under different excitation conditions. It has been shown that $1 D$ intersubband transitions can be clearly resolved under high-excitation conditions in stationary [18] and time-resolved photoluminescence experiments [16]. Comparison of PL spectra with Kohn-Luttinger type calculations taking into account the complex valence band structure in the wires [19], allowed us to identify intersubband recombination processes involving quantum wire subbands with quantum numbers as high as $n_{y}=5$ and selection rule $\Delta n_{y}=0$ $[16,18]$. However, when the optical properties are dominated by excitonic effects, as it is the case under low excitation conditions, the quantum size effects induced by the lateral confinement appear to be different. It was pointed out in Ref.[20] that in the rather wide quantum wires of our experiment the lateral confinement leads to a quantization of the excitonic center-of-mass motion, which leaves the internal structure of the exciton (i.e. the wavefunction of the relative motion of the electron and hole) unchanged and therefore 2D-like. The center-of-mass motion quantization manifests itself in the splitted heavy-hole exciton peaks of Fig.4a, which are quantized states of the translational motion of the 1 s heavyhole exciton. Therefore the linear PLE profile of Fig.4a still mantains some 2D character (including a sharp light- hole exciton resonance around $1.574 \mathrm{eV}$ ) and does not exhibit pure 1D states. The results of these experiments demonstrated 
that quantum size effects are differently pronounced under different experimental conditions. In fact, depending on the wire width $L_{y}$ one can find one-dimensional excitons if $L_{y}<a_{0}$ and two-dimensional excitons if $L_{y} \gg a_{0}$. In our sample we have roughly $L_{y} \simeq 4 a_{o}$, hence, 1 s excitons behave almost like $2 \mathrm{D}$ excitons, while $2 \mathrm{p}$ excitons, having a larger extent of the relative motion, should exhibit an almost $1 D$ character (with transition energies close to the $1 \mathrm{D}$ inter-subband transitions observed in the high-excitation intensity photoluminescence spectra of Refs. $[16,18])$.

The TPA-PLE spectra of Figs. $4 b$ and $4 c$ confirm these expectations. The spectra are different depending on the orientation of the polarization vector with respect to the quantum wire lateral quantization direction. In the $\epsilon \perp y$ configuration (Fig.4b) five structures are observed, whose relative energy splittings are the same as those observed for the $\Delta n_{y}=0$ transitions in the time resolved [16] and in the stationary luminescence spectra [18] under high photoexcitation condition, i.e. they directly reflect the 10 subband spacing. Furthermore, the first resonance of the TPA-PLE spectrum in Fig. $4 \mathrm{~b}$ is about $3 \mathrm{meV}$ below the onset of the continuum in Fig.4a. This confirms that indeed $2 p$ excitonic states are observed. The estimated binding energy of $3 \mathrm{meV}$ for the $2 \mathrm{p}$ state is additionally in good agreement with that predicted for a perfectly quantized $1 D$ exciton. In fact, such a $2 p$ exciton follows the normal $3 D$ hydrogenic series that gives for its binding energy a value of about $4 \mathrm{meV}$ in GaAs quantum wires. The spectral positions of the higher energy transitions (which are indicated by dashed lines in Fig. $4 \mathrm{~b}$ ) have been obtained by adding the $1 \mathrm{D}$ subband spacing to the transitions labelled $E_{11}^{2 p}$. The good agreement between theory and experiment confirms that the observed resonances are related to one-dimensional excitonic transitions. Therefore, the TPA-PLE peaks of Fig. $4 b$, are related to $2 p$ excited states of the $1 D$ excitons associated with $\Delta n_{y}=0$ transitions, in agreement with the theoretical expectations. In the $\epsilon \| y$ configuration the TPA-PLE spectrum appears to be even more structured, and exhibits several transitions. The vertical lines of Fig.4c represent the energy positions of the 1 s exciton states associated with $\Delta n_{y}= \pm 1$ transitions in the quantum wire. These are calculated by adding the net confinement energies of the $1 D$ conduction and valence subbands to the $E_{11}^{1 s}$ exciton energy (i.e. the detection energy). Comparison of these calculated transitions with the experimental datapoints reveals reasonable agreement. This demonstrates that in this configuration is states with $\Delta n_{y}= \pm 1$ are allowed, in agreement with the theory. However, the TPA-PLE spectrum of Fig.4c exhibits rather broad peaks in this configuration, which requires further comments. In fact, the TPA selection rules employed for the interpretation of the presented non-linear absorption data have been derived from a strict one-dimensional model $[3,7]$ which obviously should be modified to treat the intermediate regime of quasi-one-dimensional excitons where $L_{y} \simeq 4 a_{o}$. Under these conditions departures from the selection rules valid for the strictly $1 \mathrm{D}$ systems are expected due to the residual $2 \mathrm{D}$ character of the exciton wavefunctions. In particular, one should consider either pure 1D-exciton states (both 1 s and $2 p$ states) or $2 \mathrm{D}$-excitons with quantized center-of-mass motion as final states of the multiphoton absorption process. A linear combination of the 
wavefunctions of these two different excitons can be used to represent a realistic form of the quasi-1D exciton wavefunction.

\section{Conclusions}

In conclusion, we have shortly overwieved the physics of of two-photon absorption processes in quantum wells and quantum wires. Our experiments evidence the strongly anisotropic selection rules of the interband two-photon absorption related to the reduced dimensionality of the system. The excited $2 p$ excitonic states and several $\Delta n_{y}= \pm 1$ transitions have been directly studied by this non-linear absorption technique.

This work has been sponsored by the National Research Council of Italy and by the Bundesministerium für Forschung und Technologie of the Federal Republic of Germany.

\section{References}

[1] for an updated review see:

R.Cingolani and K.Ploog, Adv. Phys. 40, 535 (1991)

[2] K.Kash, J.Luminesc. 46, 69 (1990)

[3] H.N.Spector, Phys. Rev. B35, 5876 (1987)

[4] A.Pasquarello and A.Quattropani, Phys. Rev. B38,6206 (1988)

[5] A.Pasquarello and A.Quattropani, Phys. Rev. B41,12728 (1990)

[6] A.Shimizu, Phys. Rev. B40, 1403 (1989)

[7] A.Shimizu, T.Ogawa and H.Sakaki, Phys. Rev.B45, 11338 (1992).

[8] J.P. Van der Ziel and A.C.Gossard, Phys. Rev. B33, 765 (1978)

[9] D.Frhoelich, R.Wille, W.Schlapp, and G.Weimann, Phys. Rev. Lett. 61, 1878 (1988)

[10] K.Tai, A.Mysyrowicz, R.J..Slusher, and A.Y.Cho, Phys. Rev. Lett. 62, 1784 (1989)

[11] I.M. Catalano, A.Cingolani, R.Cingolani, M.Lepore, and K. Ploog, Phys. Rev. B40, 1312 (1989)

[12] I.M.Catalano, A.Cingolani, M.Lepore, R.Cingolani, and K. Ploog, Solid State Commun. 71, 217 (1989) 
[13] M.Nithisoontorn, K.Unterrainer, S.Michaelis, N.Sawaki, E. Gornik, and H.Kano, Phys. Rev. Lett. 62, 3078 (1989)

[14] I.M.Catalano, A.Cingolani, M.Lepore, R.Cingolani, and K. Ploog, Phys. Rev. B41, 12937 (1990)

[15] R.Cingolani, M.Lepore,R.Tommasi, I.M.Catalano, H.Lage, D. Heitmann, K.Ploog, A.Shimizu, H.Sakaki, and T.Ogawa, Phys. Rev. Lett. B69, 1276 (1992)

[16] R.Cingolani, H.Lage, L.Tapfer, H.Kalt, D.Heitmann, and K.Ploog, Phys. Rev. Lett. 67, 891 (1991)

[17] M.Matsuura and Y.Shinozuka, J.Phys. Soc. Japan, 53, 3138 (1984)

R.Greene , K.K.Bajai , and D.E.Phelps, Phys. Rev. B29, 1807 (1984)

L.Vina, R.T.Collins, E.E.Mendez, and W.I.Wang, Phys. Rev. Lett. 58, 832 (1988)

[18] R.Cingolani, H.Lage, H.Kalt, L.Tapfer, D.Heitmann, and K.Ploog, Semicond. Sci. Technol. 7, B287 (1992)

[19] U. Bockelmann and G.Bastard, Europhys. Lett. 15, 215 (1991)

[20] H.Lage, D.Heitmann, R.Cingolani, P.Grambow, and K.Ploog, Phys. Rev. B44, 6550 (1991)

\section{FIGURE CAPTIONS}

Fig. 1. - Energy band scheme of the two-photon absorption process. On the left, the virtual intermediate state is in the continuum, whereas on the right, the final state acts like intermediate state as well.

Fig. 2. - Logarithmic plot of the intensity dependence of the photoluminescence induced by twophoton absorption at energy $2 \hbar \omega=1.590 \mathrm{eV}$ (a) and $2 \hbar \omega=1.610 \mathrm{eV}$ (b) in the GaAs/AlGaAs quantum wires. The straight lines are the best fit quadratic regression curves to the experimental points. Curves fit all datapoints within $\pm 3 \%$.

Fig. 3. - a) OPA-PLE of the GaAs/AlGaAs quantum wells.

Fig. 4. - a) Linear photoluminescence excitation (PLE) spectrum of the quantum wire array at $\mathrm{T}=10 \mathrm{~K} . E^{c o n t}$ indicates the energy of the exciton continuum. b) TPA-PLE of the quantum wires measured in the $\epsilon \perp y$ configuration at $\mathrm{T}=10 \mathrm{~K}$. The vertical lines indicate the calculated energy positions of the $2 p$ exciton states associated with $\Delta n_{y}=0$ transitions. The horizontal error bar coincides with the full dots (the tuning accuracy was $2 \AA$ ). c) The same as in Fig. $1 b$ but in the $\epsilon \| y$ configuration. The vertical lines indicate the calculated energy positions of the 1 s exciton states associated with $\Delta n_{y}= \pm 1$ transitions $\left(E_{n_{y}, n_{y \pm 1}}^{1 s}\right)$. 


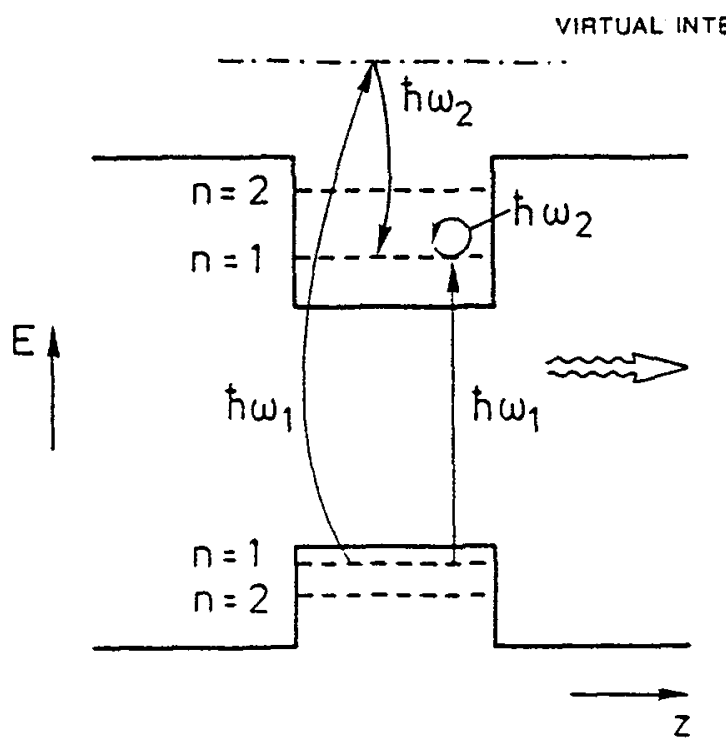

Fig. 1

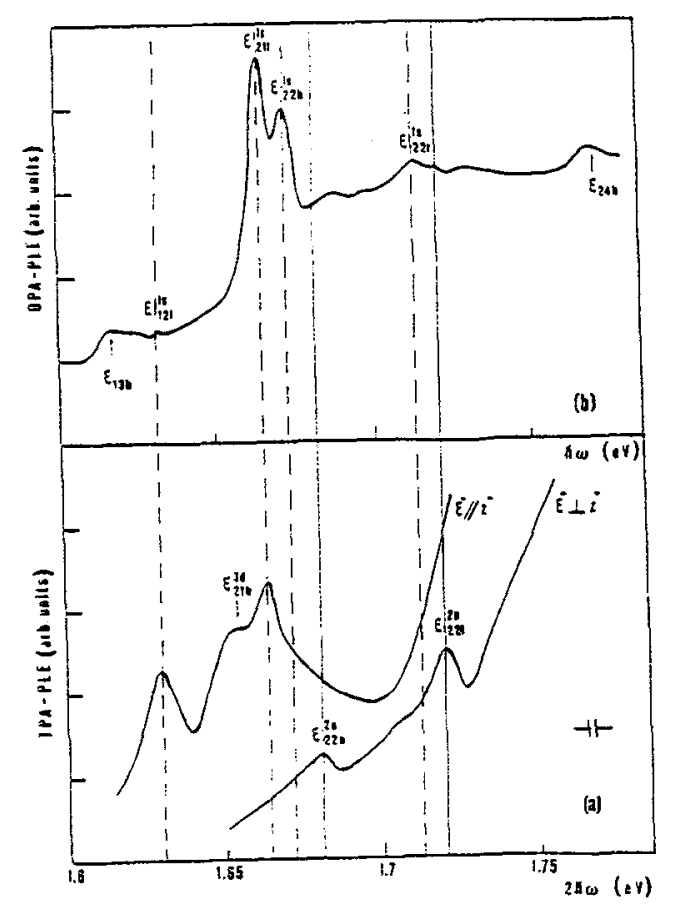

Fig. 3

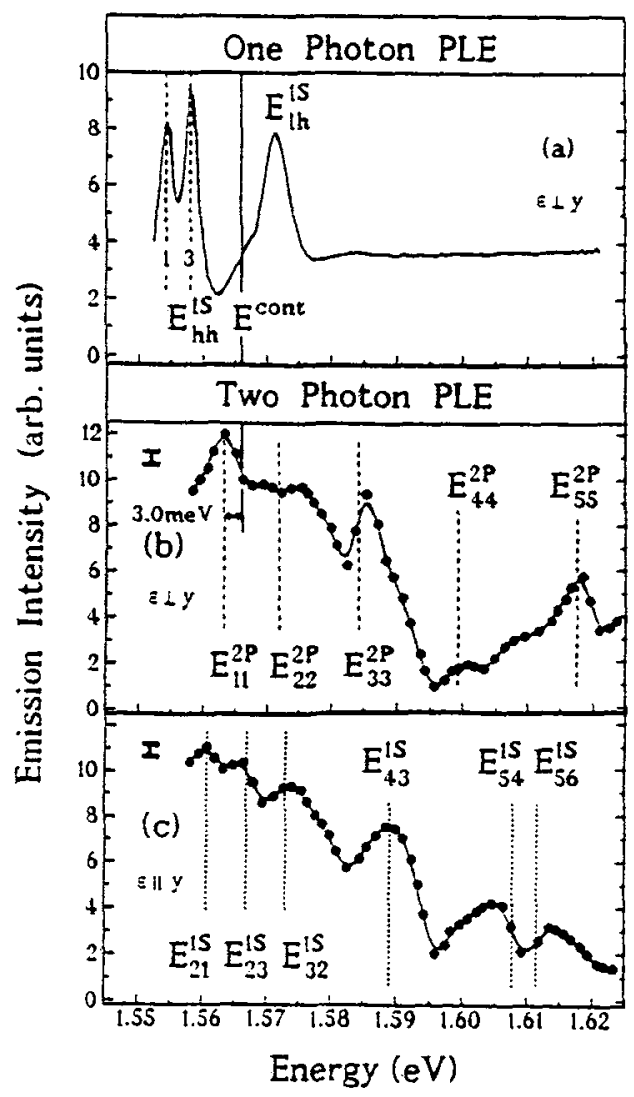

Fig. 4 